\title{
INFORMACIÓN Y DERECHOS CONSTITUCIONALES
}

\author{
SERGIO FOIS \\ Catedrático de Derecho Constitucional \\ de la Universidad de Sassari (Italia) \\ Vocal del Consejo de la Presidencia \\ del Tribunal de Cuentas de Italia
}




\section{SUMARIO}

1. El CONCEPTO Y EL FENÓMENO DE LA «INFORMACIÓN». 2. "INFORMACIÓN" Y MANIFESTACIÓN DEL PENSAMIENTO. 3. INCONSISTENCIA DE LA DISTINCIÓN ENTRE "HECHO" Y "OPINIÓN" CON RELACIÓN A LA "INFORMACIÓN". 4. ARGUMENTOS JURIDICO-CONSTITU-

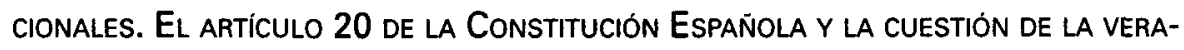
CIDAD DE LA "INFORMACIÓN". 5. LA "INFORMACIÓN" EN SENTIDO OMNICOMPRENSIVO: RAZONES, TAMBIÉN JURIDICO-FILOSÓFICAS, DE TAL OPINIÓN. 6. CRITICA DE UNA HIPOTIZABLE "CONVENCIÓN" QUE VINCULE LA INFORMACIÓN A LA "VERDAD OBJETIVA". 7. TODA "INFORMACIÓN" ES INTRINSECAMENTE "TENOENCIOSA". 8. LOS DERECHOS CONSTITUCIONALES QUE TIENEN POR OBJETO LA "INFORMACIÓN". EL DERECHO A (DE) INFORMAR COMO VERDADERO Y PROPIO DERECHO DE LIBERTAD. 9. EL DERECHO A INFORMAR - A TRAVÉS DE CUALQUiER MEDIO - NO PUEDE NUNCA SER TRATADO COMO UN PODER. 10. DERECHO A INFORMAR: PUBLICIDAD, INTERNET, Y RELACIÓN CÓN LA DEMOCRACIA. 11. EL DERECHO A RECIBIR INFORMACIÓN: SU INVIOLABILIDAD Y PERFECTA "ESPECULARIDAD" RESPECTO AL DERECHO A INFORMAR. 12. EL DERECHO A BUSCAR LA INFORMACIÓN: NECESIDAD DE SU CORRECTA DELIMITACIÓN. 13. EL DERECHO A OBTENER UNA INFORMACIÓN VERDADERA E IMPARCIAL: DUDAS SOBRE SU CONFIGURABILIDAD. LINEAS CRITICAS SOBRE EL LLAMADO "PLURALISMO"; IMPLICACIONES QUE SE DERIVAN DEL CORRECTO MODO DE ENTENDER LA "INFORMACIÓN". 14. LINEAS CONCLUSIVAS, Y ALGUNAS CONSECUENCIAS DEL DISCURSO DESARROLLADO; EL "MITO" DE LA "INFORMACIÓN" COMO "SERVICIO PÚBLICO". 


\title{
INFORMACIÓN Y DERECHOS CONSTITUCIONALES ${ }^{1}$
}

POR

\author{
SERGIO FOIS \\ Catedrático de Derecho Constitucional \\ de la Universidad de Sassari (Italia) ${ }^{2}$ \\ Vocal del Consejo de la Presidencia \\ del Tribunal de Cuentas de Italia
}

\section{EL CONCEPTO Y EL FENÓMENO DE LA «INFORMACIÓN"}

Para saber cuáles y cuántos derechos son referibles a la uinformación", y para individualizar los problemas que el discurso sobre tales derechos implica o puede implicar, hace falta necesariamente aclarar qué cosa debe entenderse, precisamente, por "información": qué cosa debe entenderse, antes que nada, desde el punto de vista conceptual y terminológico, y sucesivamente desde el punto de vista jurídico o, en todo caso, jurídico-político. Tal aclaración es necesaria a pesar de que la referencia a la «información» sea cada vez más utilizada,

1 Texto de la Conferencia pronunciada en el Curso de Doctorado sobre "Ampliaciones y minoraciones de la libertad de expresión", organizado por el Departamento de Derecho Constitucional de la UNED durante el curso académico 1999/2000. Traducción del italiano al castellano realizada por Carlos Vidal Prado, Profesor Asociado del Departamento de Derecho Constitucional de la UNED. EI texto ha sido revisado $y$ aprobado por el autor.

2 El prof. Sergio Fois ha ejercido la docencia en diversas universidades italianas. Actualmente es Ordinario en la Universidad de Sassari, y previamente lo fue en la Universidad de Roma "La Sapienza" Ha sido también miembro del Consejo Superior de la Magistratura hasta 1998. 
de modo incluso abusivo: es más, precisamente porque el término en cuestión es absolutamente difuso, la aclaración es indispensable para evitar confusiones y distorsiones en la experiencia jurídica y en el afrontamiento de los problemas inherentes a la correcta interpretación de importantes principios de los ordenamientos constitucionales liberal-democráticos.

\section{2. «INFORMACIÓN» Y MANIFESTACIÓN DEL PENSAMIENTO}

La aclaración apuntada no puede iniciarse sin preguntarnos si el fenómeno de la "información" tiene o no su propia consistencia especifica y autónoma respecto a lo que muy frecuentemente suele definirse -también en las Cartas constitucionales y entre los juristas estudiosos de la materia- como "manifestación del pensamiento". Por tal "manifestación del pensamiento» entienden los estudiosos -especialmente la mayoría de los italianos-cualquier expresión de opiniones, convicciones, actitudes, sentimientos, emociones, exhortaciones, narraciones e interpretaciones de acontecimientos de cualquier naturaleza, etc. En tal caso la enumeración realizada es meramente descriptiva y ejemplificativa, y sirve solamente para excluir que por manifestación del pensamiento pueda entenderse sólo la del pensamiento racionalmente formulado y articulado. En un intento de sintetizar lo que se considera debe entenderse por manifestación del pensamiento, probablemente puede decirse que dicho concepto se refiere al hecho de "poner de manifiesto ante otros" todo lo que tiene origen y/o consecuencia en la esfera psíquica de un sujeto determinado, y por voluntad del mismo sujeto. Me doy cuenta de que puede acusarse a la definición que acabo de expresar de excesiva amplitud, y que por eso puede considerarse, por algunos, cuando menos discutible: pero sucede que, a los fines de cuanto debe ser comprendido en la definición, lo que interesa es la voluntad de poner de manifiesto cualquier cosa que pueda ser objeto de la libertad de elección del sujeto. Entonces, la misma definición se convierte en aceptable y se revela también correspondiente a la común experiencia, así como al concepto por el cual lo que importa es que se trate de la voluntaria expresión -concretada a través de cualquier "medio" capaz de exteriorizarla - de cualquier cosa que atenga a la esfera de la personalidad de un sujeto determinado.

La mayoría de la doctrina -al menos la italiana-concluye que manifestación del pensamiento es cualquier exteriorización voluntaria, dirigida a otros, de cualquier cosa referible a la personalidad de un sujeto determinado. En este sentido incluso la manifestación del pensa- 
miento de otros es «manifestación», porque también en tal caso existe la voluntad de hacer llegar a otros contenidos "de pensamiento" que, aunque formulados por sujetos diversos, sin embargo se convierten en objeto de la voluntad de exteriorización. En realidad, el sujeto que se expresa, en un cierto sentido se apropia del contenido, en cuanto utiliza tales "objetos de pensamiento" como material para formular (para poner de manifiesto) el propio contenido comunicativo. Como ejemplo típico del perfil que acabamos de describir puede señalarse la cita de frases o posiciones de otros: es de común experiencia que la técnica de la cita sirve precisamente, a quien la utiliza, para manifestar la propia posición de "pensamiento" respecto a una cuestión determinada.

En esta misma línea es necesario hacer otra precisión previa: hasta ahora se ha hablado del "pensamiento" que se quiere poner de manifiesto para hacerlo llegar a otros: resta el problema de si se puede distinguir, y en qué términos, la manifestación dirigida a cualquiera que pueda llegar a conocerla, de la que se dirige solamente a otros sujetos determinados o determinables. El problema es, en otras palabras, el de la distinción entre la manifestación que se quiere difundir al público $y$ en público, frente a la manifestación "privada» en cuanto se quiere que llegue sólo a determinados sujetos. En términos más estrictamente jurídicos, el problema es, en definitiva, el de la distinción entre la libertad de manifestación difundida públicamente $-y$ por tanto, en este sentido, manifestación pública- y la libertad de comunicación privada; o también, utilizando la terminología del ordenamiento español, la distinción entre "comunicación social» y la comunicación sin adjetivos, que debe por tanto ser entendida como comunicación privada. Puesto que las más recientes tecnologías comunicativas convierten en problemática tal distinción - que en su momento se consideró simple-, no se trata ahora de considerar los aspectos, especialmente jurídicos, de tal distinción, y sí es oportuno, sin embargo, analizar la relación entre manifestación del pensamiento e información.

En este sentido se ha planteado si, desde un punto de vista conceptual y fenomenológico, la información es algo específico y autónomo respecto de la manifestación del pensamiento. A primera vista, y desde una perspectiva no demasiado rigurosa ${ }^{3}$, puede parecer que existe una neta y segura diferencia entre la manifestación como expre-

3 N. del T.: La expresión en italiano es la siguiente: secondo un buon senso piuttosto grossolano. Se ha traducido al castellano de un modo un tanto libre, puesto que la palabra grossolano equivale al español grosero, calificativo que no encajaba demasiado en el texto. 
sión de opiniones semejantes, por un lado, y la información como comunicación y narración de hechos, por otro, en función de lo cual estaria justificado un diverso régimen jurídico. Sin embargo, si profundizamos algo más, vemos que las cosas no son así. En realidad, en las relaciones comunicativas interpersonales todo es información, en el sentido amplio apuntado, pero al mismo tiempo nunca es sólo información en este sentido. Por una parte, cuando un sujeto expresa una opinión dirigiéndose a otros sujetos, bien puede decirse que les informa del hecho de que posee una opinión. Por otra parte, cuando un sujeto informa a otros de un determinado acontecimiento (de cualquier género que sea), lo hace imprimiendo inevitablemente su propia subjetividad sobre tal decisión informativa. A su voluntad de expresión pertenece no sólo la decisión referente al sobre qué cosa informar, sino también sobre si debe informar, al cómo informar, cuándo informar, y en qué contexto $y$ con qué relieve hacerlo.

En relación con todos los aspectos y todos los posibles matices de tal elección, el llamado "hecho" como contenido de la comunicación informativa pierde la pretensión de una objetiva, neutra y extrínseca connotación, $y$ viene en cierto modo absorbido en la esfera psíquica del sujeto que informa, convirtiéndose, en consecuencia, en manifestación de su pensamiento. En definitiva, la narración de "hechos" no es otra cosa que una de tantas descripciones y matizaciones que, y sólo a título ejemplificativo, pueden referirse al concepto de manifestación de pensamiento que, como he dicho, no puede ser otra cosa que omnicomprensivo, una vez que se percibe como imposible imitarlo a la expresión de una opinión racionalmente articulada.

Debemos partir del presupuesto, en consecuencia, de que manifestación del pensamiento e información no son otra cosa que descripciones que subrayan distintos aspectos del mismo concepto, y descripciones de un mismo fenómeno que se presenta como intrínsecamente unitario en su realidad psíquica y sociológica: esto podría ser adecuadamente explicado y confirmado si existiese la posibilidad de analizar la experiencia privada de los seres humanos y de sus relaciones, teniendo en cuenta que en la relación comunicativa, lo que se transmite y se quiere transmitir puede ser comprendido y asumido en el término de "mensaje»; esto se refiere a cualquier comunicación realizada con actitudes significativas, gestos, palabras, sonidos, entonaciones, signos y cualquier otra cosa destinada a, y capaz de, llamar la atención de otros sujetos. En este sentido, todo "mensaje» es una "información" que, también según la etimología, está dirigida a otorgar forma expresiva a cualquier cosa que un determinado sujeto quiere comunicar. 
Desde el punto de vista conceptual, fenomenológico y sociológico, la imposibilidad de atribuir fundamento adecuado a la distinción entre manifestación e información no sirve solamente entre individuos considerados en su dimensión, por así decir, privada: es útil también en el caso de que la comunicación se concrete a través de las diversas tecnologías, precisamente -en su diversidad y evolución- de los llamados medios de comunicación de masas; también en ellos toda información es siempre manifestación y viceversa. Es más, en tal caso las características tecnológicas no sólo influyen sobre el ámbito y la expresividad de la difusión comunicativa, sino también de manera significativamente variada sobre la gama de modalidades y modulaciones ofrecidas a la elección de quien utiliza dichos medios.

También en el uso de tales medios los llamados "hechos" no existen como realidad extrínseca, neutra y objetiva que justifique una adecuada distinción entre la información (como comunicación de las llamadas "noticias») y la manifestación de expresiones de convicciones subjetivas. La elección de las modalidades y las modulaciones ofrecidas por el medio permite imprimir un determinado significado expresivo a la que parecería ser simplemente la narración de una noticia. En un periódico impreso, por ejemplo, tal significado depende del título, de la colocación en una página determinada, de la colocación en el ámbito de la página, de la tipografía más o menos llamativa, además de las palabras utilizadas, de la sintaxis adoptada y de las dimensiones del medio a través del cual la noticia viene comunicada. Análoga influencia sobre el significado expresivo de lo que conocemos como noticia depende de las opciones subjetivas de quien utiliza un lenguaje de medios de comunicación más complejos, como por ejempio la radio o la televisión, porque en ellos, entre otras cosas, tal significado depende de las elecciones que se refieren a la asociación de las palabras con los sonidos e incluso de las imágenes en movimiento.

De lo expuesto hasta ahora se deriva que también en el caso de los medios impresos, de la radio, el cine, la televisión, la comunicación vía internet, no tiene sentido hablar de "información» como pura y simple narración de "hechos" extrínsecos y neutros. En realidad esto significaría proponer un significado de "información" que no sólo es inaceptablemente reductivo desde un punto de vista conceptual $y$ fenomenológico, sino que también contradice las características que el mensaje informativo inevitablemente asume a causa de las múltiples opciones expresivas intrínsecamente ofrecidas por los medios de comunicación de masas, o si se quiere de "comunicación social». 


\section{INCONSISTENCIA DE LA DISTINCIÓN ENTRE «HECHO» Y «OPINIÓN" CON RELACIÓN A LA «INFORMACIÓN"}

Me parece que la inconsistencia de cualquier tentativa de distinguir de modo adecuado la información de la manifestación del pensamiento resulta no sólo de un análisis elemental de los aspectos conceptuales y fenomenológicos de la cuestión, sino que se ve también confirmada suficientemente por lo que se ha venido afirmando muchas veces, sobre el plano jurídico, por la mejor jurisprudencia y doctrina constitucional, al menos por lo que respecta a Italia. La Corte Costituzionale, especialmente (pero no sólo) en la importantísima Sentencia 826 de 1988, ha subrayado que "siempre ha entendido la información en sentido lato y omnicomprensivo, de manera que incluye cualquier mensaje televisivo, informativo, cultural o susceptible de incidir sobre la opinión pública", así que, entre otras cosas, no seria admisible distinguir una «información en sentido técnico" (o restrictivo, o reductivo), incluso cuando se trata de "programas de puro espectáculo", "de entretenimiento o de ocio". El elemento más significativo ofrecido por esta jurisprudencia constitucional debe ser individuado en la sintética afirmación según la cual, para comprender el objeto de la correspondiente libertad constitucional, lo que interesa es considerar como "información" cualquier manifestación, concretada a través de cualquier medio (también el televisivo), que sea capaz de incidir sobre la opinión pública: según mi criterio, esto significa que también aquello que en apariencia incluye la pura y simple narración de un determinado "hecho", en realidad implica e incluye la comunicación de cualquier cosa que viene elaborada en la esfera psíquica de un sujeto agente, y que de cualquier manera es intrínsecamente capaz de producir efectos en la esfera psíquica de los sujetos destinatarios del mensaje.

En la misma lógica que excluye la practicidad jurídica de una distinción entre "hechos" y "opiniones", y en consecuencia necesariamente de una distinción entre manifestaciones e informaciones, se enmarca, por ejemplo, el pensamiento de uno de los mayores constitucionalistas italianos, Barile, quien afirma muy claramente que "separar el hecho del juicio (por ejemplo, según el modo como se les presente), es, según la común y pacífica experiencia (...) prácticamente imposible, al menos en la mayoría de los casos". Creo que, en términos generales, la posición de la doctrina constitucional italiana ha sido expresada de modo correcto, y muy recientemente (en 1998), por Zaccaria, el cual afirma que, "considerando (...) el lado activo de la libertad de expresión o de información", debe "establecerse una equi- 
valencia entre el derecho de manifestar, el derecho de informar y el derecho de crónica»; de esto se deriva que "no estamos frente (...) a distintos derechos, sino a una única libertad", cuyo objeto se contiene y está enteramente comprendido y garantizado en el art. 21 de la Constitución italiana.

\section{ARGUMENTOS JURÍDICO-CONSTITUCIONALES. EL ARTÍCULO 20 DE LA CONSTITUCIÓN ESPAÑOLA Y LA CUESTIÓN DE LA VERACIDAD DE LA «INFORMACIÓN»}

Cuanto resulta respecto al correcto modo de entender, también en el plano jurídico, el fenómeno y el concepto de "información", me ofrece elementos para nutrir una notable perplejidad al confrontarlo con la formulación que parece acogida en el artículo 20 de la actual Constitución española, según la cual subsistirían dos diversos y distintos derechos constitucionales: el primero, a expresar y difundir libremente los pensamientos, ideas y opiniones; y el segundo, por el contrario, a comunicar o recibir libremente informaciones veraces a través de cualquier medio de difusión. Frente a semejante formulación me parece difícil admitir que la referencia a los dos derechos quiera tener un valor de elenco simplemente descriptivo. Efectivamente, no sólo los términos utilizados son absolutamente diversos, de manera que aluden a una supuesta distinción neta entre las "opiniones" y los "hechos", sino que además se pone en evidencia el problema de aquéllo que en definitiva debería representar el requisito más importante de lo que conocemos como "información", y en consecuencia el límite principal del correspondiente derecho, un límite que resulta esencial e intrínseco: el de la "verdad" de las "informaciones", de forma que la garantía constitucional del derecho en cuestión ampararía solamente las informaciones "veraces".

Frente a la fórmula del art. 20 de la Constitución española, la cuestión de la distinción o no entre manifestación e información asume un significado no sólo conceptual y terminológico, sino que incluso puede llegar a plantear implicaciones concretas y muy relevantes en el plano jurídico. Se pretende, en definitiva, distinguir la información de la manifestación para circunscribir la primera a la mera comunicación de hechos totalmente objetivos y en todo caso extraños a la esfera psíquica del sujeto comunicante: la información sería tal solamente cuando subsistiese una plena e integral correspondencia con la objetiva y externa realidad del "hecho" asumido como objeto de la comunicación informativa. Semejante correspondencia equivaldría automáti- 
camente a la "verdad" de la misma información, de forma que tal verdad sería, al tiempo que característica intrínseca, el límite necesario de una información que podría considerarse constitucionalmente garantizada sólo y en cuanto resulta ser efectivamente "verdadera".

Como es evidente, el citado modo de entender la información da por descontado que la verdad objetiva de los hechos comunicados debe ser una característica intrínseca de los principales aspectos de la actividad desarrollada por los medios de comunicación de masas (o si se quiere, de comunicación social), de modo que tendría respuesta netamente afirmativa la cuestión, notablemente debatida en las diversas experiencias jurídicas, de si el llamado derecho de crónica encuentra siempre su límite en el respeto de una verdad absolutamente objetiva o si, por el contrario, esto representa un pretexto injustificado precisamente porque un respeto semejante es conceptual y prácticamente imposible. En el primer caso, el derecho en cuestión debería reconducirse hacia una libertad de información bien distinta a la manifestación de ideas, opiniones, etc.; en el segundo caso, todo lo más, el derecho de crónica puede encontrar un límite solamente en la "verosimilitud" de los hechos narrados, y por tanto solamente en una "verdad putativa" en cuanto que valorada como razonable por el sujeto que comunica la información: en definitiva, en una verdad sobre la que el sujeto en cuestión se considere de algún modo autorizado a considerarla como tal.

La consecuencia es que en el primer caso, la información se separa casi totalmente (salvo en lo que se refiere a la elección sobre si comunicar algo o nol de la esfera psiquica subjetiva de quien efectúa la comunicación, en tanto que tiene por objeto un "hecho" que, en cuanto que objetivamente verdadero, por sí mismo debería conducir al sujeto que comunica a prescindir de la actitud personal que ha adoptado al confrontarlo. En el segundo caso, sin embargo, la información no sería otra cosa, todo lo más, que un tipo de modalidad de la manifestación narrativa, y el llamado "hecho" sería siempre cualquier cosa que existe sólo en cuanto que se asume como propia y (por asi decir) se recrea por la (y en la) esfera psíquica subjetiva del sujeto comunicante.

5. LA «INFORMACIÓN" EN SENTIDO OMNICOMPRENSIVO: RAZONES, TAMBIÉN JURIDICO-FILOSÓFICAS, DE TAL OPINIÓN

Como intentaré explicar, las alternativas que acabo de indicar tienen implicaciones y consecuencias relevantes sobre una serie de 
cuestiones que afectan a la problemática jurídico-constitucional en el ámbito de la información. Pero, antes de señalar las que considero como más relevantes entre tales consecuencias, deseo motivar todavía por qué mi opción se ha orientado en el sentido de considerar la información como absolutamente omnicomprensiva, $y$ por ello también como manifestación plena de una elaboración que tiene lugar en la esfera psíquica del sujeto que la comunica, sin que la misma encuentre un límite rígido y seguro en la realidad objetiva de los hechos, que se presume del todo externa e independiente de dicha esfera. Los motivos de mi posición se derivan no sólo de cuanto se puede deducir del sumario análisis que anteriormente he tratado de desarrollar sobre el plano conceptual y fenomenológico, sino también de ulteriores argumentos que toman causa en las recientes orientaciones de la (para mí) mejor filosofía epistemológica. Aquél que mejor ha formulado y sintetizado tales orientaciones es, a mi juicio, Popper, que más que cualquier otro se ha dedicado a analizar el problema del conocimiento, especialmente de las ciencias, incluyendo las experimentales y las llamadas "exactas", en orden a la posibilidad o no de concebir una estrecha y definitiva correspondencia entre los objetos del aprendizaje científico, entre los "datos" que la realidad externa parece ofrecer, y aquéllo que el sujeto puede alcanzar a conocer $y$ aseverar con relación a tales objetos y datos. En su "Lógica del descubrimiento científico", Popper afirma que el conocimiento empírico, también el científico, "no tiene en sí mismo nada de absoluto", de forma que "las mismas afirmaciones básicas" (esto es, "en resumen; la aseveración de un hecho singular») "no son justificables sobre la base de nuestra experiencia inmediata». Conviene subrayar que Popper entiende por "afirmación singular (una afirmación básica)" aquella que "describe un acontecimiento"; es por tanto evidente la estrechísima analogía entre el caso de la afirmación científica y la comunicación informativa de un "hecho" determinado: pero, así como la afirmación científica básica no puede - según el filósofo- ser demostrada como verdadera, sino todo lo más falsificada, otro tanto es posible decir para la comunicación informativa, de la cual no es por tanto admisible pretender su intrínseca e indiscutible veracidad.

En síntesis, según las orientaciones de la mejor filosofía del conocimiento, la aceptación y la comunicación de hechos determinados no pueden ser ancladas en una inalcanzable y vinculante verdad objetiva. Se podria objetar que es injustificado aplicar a los fenómenos de la comunicación social cuanto se retiene válido en el plano de una filosofía del conocimiento, mucho más sofisticada, máxime si se pretende aplicar tal analogía al campo de la experiencia jurídica, que tie- 
ne su propia especificidad y autonomía. Al mismo nivel, sin embargo, se puede responder primeramente recordando que Popper afirma con decisión que "el conocimiento científico no es otra cosa que un desarrollo del conocimiento ordinario, o conocimiento del sentido común". De manera que, cuanto se considera válido para el primero no puede no ser válido para el segundo, y viceversa. En segundo lugar se puede hacer notar que precisamente Popper, para explicar por qué las afirmaciones básicas no son justificables sobre la base de nuestras experiencias inmediatas, utiliza expresamente la analogía del "proceso con jurado", porque "como el del que experimenta, el veredicto del jurado (vere dictum: dicho con verdad) es la respuesta a una cuestión de hecho". La filosofia de Popper, al criticar cualquier pretensión positiva de verdad objetiva, tiene por tanto bien presente cómo esto debe valer con referencia a lo que se orienta sobre el plano del sentido común y sobre lo que ahora más nos interesa, la experiencia jurídica.

A favor de mi convicción en lo que se refiere a la imposibilidad de delimitar la información a la sola exposición de hechos verdaderos - puesto que la verdad de tales hechos no puede nunca darse por descontada ni en el momento en el cual se le conoce ni en el cual en que se comunica一, no sólo está la enseñanza de la mejor filosofía, sino también argumentos evidenciables bien a través del significado propio de importantes obras de arte, o por medio de ejemplos que ofrecen significativos experimentos en el campo socio-jurídico. A tal fin recuerdo por un lado el film Rashomon y el teatro de Pirandello, y por otro lado cuanto ha resultado de experimentos basados en testimonios "oculares": en ambos casos se muestra con particular fuerza que un mismo y presuntamente idéntico hecho es objeto de modo inevitable de descripciones profundamente diversas e incluso contradictorias.

Existe todavía otro posible modo de replantear la objeción más arriba apuntada, que contrasta con el (mi) convencimiento de considerar inaceptable cualquier pretensión de distinguir netamente los "hechos" de las "opiniones", y de confinar el concepto de información a la sola y "verdadera" narración de "hechos". Se podría advertir que, si bien la inaceptabilidad tiene un fundamento en el plano filosófico y (por así decir) sociológico, con el refrendo ofrecido por significativas expresiones artísticas y experimentos concretos, sin embargo la especificidad de la información y su intrínseca vinculación con la "verdad" de los hechos asumiría un necesario sentido y una adecuada plausibilidad si se considerase que sobre el terreno jurídico fuese posi- 
ble, y efectivamente exista, una forma de "convención" según la cual es oportuno que se piense y se actúe en términos de verdad objetiva, de modo que esto pueda hacerse valer en las relaciones jurídicas $y$, en particular, en la relación que se establece con la comunicación informativa. Tal "convención" plantearía la exigencia de la verdad en el caso de la comunicación de "hechos", y de distinguir la información de la opinión, a pesar de que todo ello sea por muchas razones imposible en el plano extrajurídico.

\section{CRÍTICA DE UNA HIPOTIZABLE "CONVENCIÓN" QUE VINCULE LA INFORMACIÓN A LA "VERDAD OBJETIVA»}

Sin embargo, una convención semejante -que en definitiva violenta la naturaleza de las cosas si se considera a nivel filosófico, sociológico y filosófico- puede ser invocada sólo en casos y bajo perfiles no definidos, y por lo tanto no en el caso de la comunicación informativa. Efectivamente, tal convención, inspirada en exigencias no contingentes de política del derecho, puede justificar, por ejemplo y de modo especial, que el ordenamiento jurídico imponga la necesidad de hacer valer como definitivamente "verdaderas" las decisiones tomadas en sentencias firmes o irreversibles, y también de considerar verdaderas de modo irrechazable las interpretaciones normativas y las reconstrucciones de hechos realizadas precisamente en las sentencias ahora señaladas. En los casos citados la convención tiene, en definitiva, un sentido aceptable, porque se trata de conseguir que mecanismos esenciales del ordenamiento jurídico funcionen de modo que se intente asegurar el valor de la certeza del derecho al cual es difícil renunciar. Pero, y en primer lugar, en los casos ahora indicados, la "verdad" que se acuerda hacer valer es meramente "procesal", esto es, sólo aquélla que resulta método lógicamente del desarrollo del procedimiento jurisdiccional. No se puede albergar la pretensión de considerarla como verdad "sustancial" efectiva y objetiva, porque se trata de una verdad impuesta, en nombre de la certeza, por efecto de un acto voluntario $y$ de autoridad.

En segundo lugar, es posible afirmar que puede tener sentido la validez de la "convención de la verdad" sólo para aquellos tipos y aspectos de relaciones jurídicas en las cuales debe prevalecer la exigencia de una certeza en orden a situaciones y evoluciones coyunturales que resultan relevantes para asegurar en cualquier modo la previsibilidad $y$ orden de la ( $y$ en la) vida en sociedad y en los comportamientos de sus instituciones. La imposición de una verdad meramente proce- 
sal, y para ciertos aspectos también de una determinada interpretación normativa, tiene por objeto, y por excelencia, tal específica finalidad. La convención que conduce a la imposición de una "verdad" semejante no tiene, por el contrario, sentido y justificación en el caso de que se trate de otros casos y aspectos de las relaciones intersubjetivas: para ellas la presuposición de una tal forma de verdad no se muestra necesaria y oportuna, es más, resulta tal vez contraindicada en función de la naturaleza y el significado de la relación considerada. Es éste el caso de la relación que se establece por la ( $y$ en la) comunicación informativa, donde la exigencia de la libertad de las actitudes puede prevalecer sobre la de una certeza por lo demás necesariamente impuesta. En breves palabras: la afirmación de la existencia del contenido de derechos y obligaciones, así como la de la existencia y el contenido de una pretensión sancionatoria y de un correspondiente sometimiento punitivo, exigen una certeza que en definitiva debe poder cristalizarse en una "verdad procesal".

Sin embargo, en el caso de la relación informativa, las exigencias se disponen en un modo que tiende a ser diametralmente opuesto, porque en dicha relación se privilegia intrínsecamente el principio de la libertad de quien informa de igual manera que el de la libertad de quien es informado. En general me parece que, efectivamente, puede decirse que la verdadera esencia de la libertad está en la duda, y por tanto, en el exacto contrario de cualquier forma de certeza, especialmente si ésta debe ser autoritariamente impuesta. La libre elección en el marco de las dudas de diverso nivel y relieve debe ser situada en la esfera psíquica del sujeto que efectúa la comunicación informativa así como en la del sujeto que la recibe. En síntesis, mientras en el caso de la determinación de los derechos y obligaciones es necesario que exista la certeza, incluso en ausencia de una correspondencia con la "verdad sustancial» precisamente para que se pueda recurrir en última instancia a la "verdad procesal", en el caso de la relación informativa, por el contrario, no debe exigirse esa certeza aunque (pero sólo en apariencia) exista la posibilidad de invocar la correspondencia con una "verdad sustancial" y objetiva, aunque sólo aparente. Se puede añadir además que, mientras en el primer caso estamos en el campo de derechos $y$ obligaciones cuya afirmación implica la producción de efectos jurídicos específicos y bien cualificados en la esfera jurídica de otros sujetos -lo que justifica la imposición de una certeza vinculada a la "verdad procesal"-, en el caso de la relación informativa nos encontramos en el campo de situaciones jurídicas (la de quien informa y la de quien es informado) cuyo goce no implica generalmente la producción de algún efecto jurídico en los sujetos interesados y en terceros sujetos. Preci- 
samente por razón de la producción de los efectos jurídicos que acabamos de mencionar en el primer caso se justifica la "convención" que conduce a la imposición autoritaria de cualquier "verdad", mientras en el segundo caso, al faltar la producción de cualquier efecto jurídico, una análoga "convención" resulta del todo injustificada y por lo tanto inaceptable.

Las consideraciones precedentes tienen el sentido de confirmar que ni siquiera una "convención" semejante puede efectivamente justificar una distinción apreciable entre información y manifestación, ni puede imponer a la manifestación informativa un vínculo de respeto hacia una verdad objetiva que en realidad no es nunca concebible $y$ configurable como tal. Una vez más se confirma, por tanto, que la llamada "información" no es otra cosa que la verdadera y propia manifestación del pensamiento, y en consecuencia no puede dejar de implicar todas las inclinaciones, acentuaciones $y$ elecciones que inevitablemente condicionan y caracterizan la formación del pensamiento del sujeto que expresa cualquier tipo de manifestación, también la manifestación informativa. El tratamiento jurídico de la «información", por ello, debe ser en todo y por todo idéntico al de cualquier tipo y forma de manifestación: su régimen jurídico no puede implicar "privilegios", sean positivos o negativos, de carácter especial, con la consecuencia, antes que nada, de que en sus aplicaciones concretas no puede tener sentido el límite especial del respeto a la "verdad objetivan, además de que el conocido como derecho de crónica, especialmente el del periodista, no puede ser considerado y configurado también como un «deber».

\section{TODA «INFORMACIÓN» ES INTRÍNSECAMENTE "TENDENCIOSA"}

De todo cuanto he intentado decir se deduce, además, que toda manifestación informativa es, por sí misma, inevitable e intrínsecamente, de alguna manera "tendenciosa", en el sentido etimológico del término, por lo que la misma nace y se expresa con la impronta de las motivaciones $y$ de las intenciones propias del sujeto que la hace efectiva. Precisamente por su intrínseca "tendenciosidad" la información puede asumir perfectamente el aspecto de la apología y la propaganda, manifestaciones que pueden ser reconducidas a la voluntad de imprimir acentuaciones unilaterales, e incluso exageraciones, dirigidas a conseguir una particular eficacia y la capacidad de incidir sobre la opinión pública que la Corte Costituzionale italiana considera, como se ha 
visto, el común denominador que asemeja todo tipo y forma de mensaje informativo. Bajo el perfil jurídico, asociar la tendenciosidad de la información a la apología y la propaganda, trae como consecuencia que, allí donde se consideren tales formas de información como legítima expresión de libertad - tal y como sostienen efectivamente la mayoría de los estudiosos italianos-, también las informaciones «tendenciosas" deben ser consideradas por sí mismas legítimas: de ello parece derivarse, por ejemplo, la ilegitimidad constitucional de la norma penal italiana (artículo 656 del Código penal) que pretende vetar las noticias falsas, exageradas o tendenciosas.

\section{LOS DERECHOS CONSTITUCIONALES TIENEN POR OBJETO LA «INFORMACIÓN». EL DERECHO A (DE) INFORMAR COMO VERDADERO Y PROPIO DERECHO DE LIBERTAD}

Cuanto se ha tratado de analizar con relación al correcto modo de entender la información y a la cuestión de si la "verdad objetiva" deba representar una característica intrínseca y un límite específico de dicha información, permite considerar de modo adecuado la cuestión de cuáles y cuántos derechos constitucionales son referibles a la información misma. La doctrina más reciente, especialmente la italiana, sobre la base también de varios pronunciamientos de la jurisprudencia constitucional, habla habitualmente de los siguientes derechos. En primer lugar, y es obvio debido a la necesaria referencia al primer apartado del artículo 21 de la Constitución italiana, se subraya la existencia ly la garantía) del derecho de cualquiera a comunicar a otros cualquier tipo y forma de información, naturalmente dentro de los límites que pueden imponerse a la correspondiente libertad de acuerdo con la Constitución. En segundo lugar, se habla del derecho a recibir las informaciones que se hagan efectivas en el ejercicio del primero de los derechos ahora mencionados. En tercer lugar se habla también, si es posible distinguirlo con una propia especificidad, del derecho a buscar las informaciones que puedan revestir cualquier interés para el sujeto que pueda ser considerado titular de ese derecho. En cuarto lugar, finalmente, algunos estudiosos hablan del derecho de obtener informaciones que tengan un determinado tipo de contenido $y$ determinadas características.

En general se considera que el primero de los derechos mencionados afecta al lado "activo", por asi decir, de la información. Sin embargo, se entiende que el segundo de los derechos en cuestión afecta al lado "pasivo" de la relación de la comunicación informativa. Por 
lo que se refiere al tercero de tales derechos, se considera mucho más dudoso que se sitúe, $y$ en cualquier caso no de modo exclusivo, en el lado "pasivo" de la relación ahora mencionada. El cuarto derecho, finalmente, dejando aparte las dudas sobre su existencia y configurabilidad, me parece que prescinde de su colocación "activa» o "pasiva" en el ámbito de dicha relación informativa: efectivamente, el mismo parece implicar la exigencia de formas de obligación a cargo de los sujetos (todos o al menos algunos) que difunden la información.

El primero de los derechos, el de comunicar cualquier tipo y forma de información, es el más tradicional y, por así decir, "clásico». Es el que ha sido objeto de los más antiguos reconocimientos constitucionales, especialmente bajo el perfil de la libertad de manifestar las convicciones y las disposiciones en materia religiosa, de la libertad de imprenta, de la libertad de comunicar las propias producciones artisticas y científicas. Actualmente es un derecho que se reconoce frecuentemente de modo muy general, con fórmulas referidas, o interpretables como referidas, a cualquier "manifestación del pensamiento", especialmente si se dirige a conseguir fines que puedan parecer (pero que no son nunca los únicos) «informativos". Muchos, yo entre ellos, entienden que se trata de un "derecho de libertad", en el sentido estricto y riguroso del término y por lo tanto, que presenta, jurídicamente, una estructura y validez que debe definirse como "negativa", en el sentido de tener por objeto una "libertad frente a" ${ }^{4}$, de modo que excluye en su esfera intervenciones externas que limiten o impidan el goce de la libertad misma. En cuanto derecho de libertad en el sentido apuntado, forma parte de aquellos derechos que se distinguen y se contraponen a los llamados "derechos sociales", que tienen por objeto la pretensión de obtener una prestación: esta neta distinción entre las dos categorías de derechos ha sido subrayada muchas veces, por ejemplo, por Calamandrei y Bobbio. Precisamente por su estructura y validez jurídicamente negativas, el derecho en cuestión no puede ser configurado y calificado, de ningún modo, como "funcional». En su esencia, este derecho, como todos los de libertad, no debe responder a otro objeto que el de consentir la expresión de los aspectos psíquicos de la personalidad de quien es titular. Los perfiles ahora

4 N. del T.: En el texto original se utiliza la expresión «libertá da», que se contrapone a «libertà di». La primera se refiere a la libertad de informar en sentido negativo, es decir, la que garantiza un espacio libre cuyos límites no pueden ser sobrepasados por los poderes públicos. Se trata de una libertad "frente» a la actuación de los poderes públicos. La segunda expresión se refiere al sentido positivo de la libertad de informar. 
indicados explican por qué el derecho en cuestión debe necesariamente amparar también la pretensión jurídica de no manifestar el propio pensamiento, y por tanto a no comunicar cualquier pretendida información: en otros términos, ninguno puede ser forzado a proporcionar las informaciones que no quiere comunicar, y por tanto, como implicación lógicamente necesaria, a proporcionar informaciones con modalidad y contenidos que no dependan de su libre elección. Es más, el derecho a informar como pura y simple modalidad de manifestación del pensamiento, y por las razones y bajo los perfiles antes apuntados, debe ser jurídicamente considerado como "inviolable", y por consiguiente formando parte de aquellos "principios supremos" del ordenamiento a los cuales la Corte Constitucional italiana ha hecho expresa referencia.

\section{EL DERECHO A INFORMAR - A TRAVÉS DE CUALQUIER MEDIO- NO PUEDE NUNCA SER TRATADO COMO UN PODER}

Todas las características del derecho individual a informar convergen en el sentido de rechazar la tendencia de algunos, también de los especialistas, para los que la "información" no deberia ser considerada tanto una "libertad", como por el contrario un "poder», que en particular sería tal cuando dicha información fuese difundida a través de medios que posean una acentuada fuerza de sugestión y persuasión; lo cual se verificaría en el caso de la radio, y todavía más de la televisión. Es preciso ante todo resaltar cuanto se ha dicho ya, en función de lo cual cualquier manifestación, asuma o no la modulación informativa, no puede no concretarse en un determinado medio; y la elección del medio, también en relación con su potencialidad difusora de la manifestación, es inherente del todo y en todo a la libertad de elección que se traduce en esa manifestación más que en otras formas y modalidades de expresión. Bajo este perfil no parece justificado distinguir, también y especialmente desde el punto de vista jurídico, la manifestación, por un lado, del "medio" utilizado, por otro. No tanto y no sólo para difundirla cuanto, de modo diverso, para concretarla y, por así decir "materializarla" hacia el exterior de la propia esfera psíquica interna. Desde esta perspectiva, como puede verse, tiene poco sentido, también jurídicamente, distinguir la libertad de manifestación de la libertad en el uso de los medios de difusión: la elección de un medio determinado y de sus capacidades expresivas y comunicativas no puede alcanzarse más que en razón de las características ofrecidas por un determinado "medio" y de las modalidades "sociales" y tecnológicas 
propias de tal medio. Una manifestación, también la informativa, no puede no encarnarse en una determinada "materia" (del modo como sucede, con particular evidencia aunque no solamente, en la producción artística). Dicha "materia» es precisamente el "medio» que se ha elegido $y$ en el sentido en el cual se ha elegido. La manifestación -también la informativa- y el medio son objeto de una sola elección, y por tanto de una única libertad. Por otra parte es obvio y del todo natural que el sujeto que pretende manifestar algo puede elegir el medio que considera más adecuado para "materializar» la expresión, porque lo considera sociológicamente más eficaz y tecnológicamente más perfeccionado. Por ello, debe admitirse plenamente que la alternativa es o la de considerar "poderes" tanto los medios de "difusión", comenzando por la palabra más o menos amplificada, hasta alcanzar a las más sofisticadas tecnologias televisivas; o, por el contrario, no considerar "poderes" ninguno de tales medios. En la primera alternativa resultaría quebrado en su raíz el mismo principio de libertad en cuanto referido o referible a cualquier medio difusor de comunicación, también aquéllos menos recientes y tecnológicamente más simples: incluso la imprenta, por tanto, que en su tiempo representó una gran revolución a nivel tecnológico y socio-político. En la segunda alternativa, sin embargo, cualquier medio de difusión debe ser considerado, y jurídicamente tratado, como objeto de una verdadera y propia "libertad» y, por tanto, debe serlo en conformidad con la naturaleza y las características de un derecho clásico de libertad: derecho que con razón se entiende no implica el ejercicio de un poder, sino por el contrario, la defensa frente al poder.

Contra la tendencia a considerar el uso de los medios de difusión jurídicamente como un poder está además la consideración, por un lado, de que la información, en cuanto que no es nunca pura y simple comunicación neutral de "hechos", sino por el contrario siempre de algún modo "tendenciosa", implica que se haga efectiva también por razón de la capacidad sugestiva del medio en el cual se concreta el "mensaje"; por otro lado se encuentra la consideración de que juridicamente no se puede de ningún modo confundir la capacidad de influencia que todos los derechos (y no sólo el de la información) pueden implicar a nivel puramente sociológico, con los bien precisos $y$ específicos efectos jurídicos a cargo de terceros que sólo un verdadero y propio "poder» puede implicar e implica. En conclusión sobre este punto, el derecho a "informar", también y especialmente en sentido omnicomprensivo, desde un punto de vista juridico constitucional debe ser siempre considerado y regulado como una "mera libertad" y no por el contrario como un "poder jurídico". 


\section{DERECHO A INFORMAR: PUBLICIDAD, INTERNET Y RELACIÓN CON LA DEMOCRACIA}

Cuanto hasta ahora he pretendido decir respecto a la información entendida en sentido omnicomprensivo, a la libertad en la elección de los fines que la misma puede estar dirigida a perseguir, al hecho de que la información pueda expresar acentuaciones propagandísticas, a su natural e intrínseca "tendenciosidad"; todo ello sirve para plantear problemas muy actuales como los que se refieren a la publicidad, también la económica y comercial. Considero que la información publicitaria debe considerarse información a todos los efectos, y por tanto debe ser considerada y garantizada como objeto del derecho de libertad del cual estamos hablando. En particular, semejante presupuesto me parece que debe servir para situar correctamente las debatidas cuestiones relativas al tratamiento jurídico de los "spots" de publicidad política y electoral: ¿por qué razones dichos "spots" no podrian ser considerados una de tantas concreciones que el derecho de libertad de informar puede legítimamente implicar?

En relación con el primero de los derechos más arriba apuntados relativos a la "información", resta decir alguna cosa en cuanto al fenómeno por el cual, actualmente, a causa de las tecnologías interactivas y en particular de internet, tiende cada vez más a desaparecer la tradicional distinción entre la información como comunicación hacia una esfera de destinatarios indeterminados o indeterminables, $y$ la comunicación dirigida a singulares y determinados destinatarios, esto es, la que viene calificada habitualmente como comunicación "privada". Al respecto, muy recientemente y de modo acertado, se ha dicho que, como consecuencia de la cada vez más rápida evolución tecnológica antes apuntada, y con relación a la libertad de difusión tutelada en Italia por el artículo 21 de la Constitución y a la libertad de comunicación y correspondencia protegida por su parte por el artículo 15, "un mismo tipo de comunicación presenta tal vez las connołaciones de ambas libertades (determinación de los destinatarios y capacidad de difusión del mensaje)", y "cómo todo ello hace siempre más difícil, además de reductivo, hablar de que un medio concreto está destinado a la comunicación interpersonal más que a la manifestación del pensamiento». Las nuevas tecnologías, por tanto, parecen permitir a los sujetos individuales, y por lo demás de manera que puede ser interactiva, la modulación del uso del medio de comunicación de modo que el mismo "mensaje" puede ser a la vez o alternativamente información para el público $\mathrm{y} / \mathrm{o}$ información "privada»; todo ello dentro de ciertos límites justifica que se pueda hablar de una úni- 
ca libertad de comunicación, que comprende la libertad de manifestación y la de comunicación interpersonal. Pero, si la tendencia es la de fundir en un único derecho los hasta ahora garantizados de modo diverso por el artículo 21 y el artículo 15 de la Constitución italiana, esto trae consigo de un lado la confirmación de que el derecho a informar tiene una naturaleza absolutamente inviolable, y de otro lado excluye definitivamente la posibilidad de considerar y tratar como un "poder" la libertad de informar, que puede tomar la forma también de la libertad de correspondencia privada. Esto contribuye a hacer caer también el último "totem" al cual se aferran todos aquellos que devalúan el valor de la libertad de información como derecho exquisitamente individual, no funcional ni funcionalizable, y que tienden a someterlo a cláusulas generales basadas en la referencia a intereses que trascienden los del individuo singular.

En la cuestión del derecho a informar, un último aspecto se refiere a su relación con la democracia, obviamente aquélla propia de un Estado de Derecho y uliberal». A pesar de que los flujos y la dialéctica informativa sirvan para hacer posible una formación articulada de la opinión pública y por tanto consientan un libre y consciente ejercicio de los derechos políticos y el efectivo funcionamiento de las instituciones democráticas, Bobbio ha subrayado que el derecho a informar es, propiamente, solamente una condición previa de la democracia, y no una componente suya exclusiva y específica. Por mi parte, he tenido ocasión de poner de relieve que, mientras que la democracia no es ni concebible ni practicable sin el goce del derecho en cuestión (junto al de los demás derechos de libertad), no es cierto sin embargo a la inversa: histórica, política y conceptualmente el derecho a la manifestación informativa puede ser perfectamente concebible y practicable incluso en ausencia de una verdadera y propia democracia. En una perspectiva semejante, se debe excluir que el derecho a informar pueda ser limitado y regulado para asegurar un presunto mejor funcionamiento de las instituciones democráticas, o que pueda considerarse funcionalizado o funcionalizable a beneficio de tales instituciones. En la misma perspectiva seria necesario analizar la cuestión del "pluralismo" y la de su valor y significado. El principio del "pluralismo", especialmente si se entiende de modo que siempre es genérico y ambiguo, no puede ser considerado un "valor" que trascienda el interés del individuo singular para comunicar, cómo y cuándo considere más oportuno, las informaciones. El pluralismo puede ser solamente el resultado del pleno goce de la libertad individual, y no una condición, por lo demás limitativa, de tal goce. 


\section{EL DERECHO A RECIBIR INFORMACIÓN: SU INVIOLABILIDAD Y PERFECTA ESPECULARIDAD RESPECTO AL DERECHO A INFORMAR}

Breves palabras ahora sobre el segundo de los derechos que precedentemente he indicado, esto es, el derecho a recibir informaciones. Este derecho algunas veces no se menciona expresamente en las fórmulas constitucionales, tal como se verifica en Italia, otras veces sin embargo se menciona, como por ejemplo sucede en España y en algunos acuerdos internacionales. La cuestión es si la existencia o no de tal expresa mención es relevante con relación a la garantía constitucional del derecho en cuestión. Mi respuesta es decididamente negativa. Para llegar a tal conclusión debo hacer notar que no es necesario recurrir al principio por el cual la garantía de la libre manifestación informativa implicaría indirectamente el reconocimiento del derecho en cuestión, en cuanto que la libertad de informar encontraría su razón, o al menos una de sus razones, en el interés de todos, o en el interés general (que no es la misma cosa) a ser informados; es ésta la vía que parece ha seguido la jurisprudencia de la Corte Costituzionale italiana. En mi opinión, por el contrario, la garantía del derecho a informar incluye, implícitamente, pero de manera absolutamente necesaria y por asi decir automática, el derecho a recibir las informaciones, precisamente porque a tal fin, y sólo a tal fin, esas informaciones son voluntariamente comunicadas. En otros términos, el derecho a informar no tendría ni siquiera sentido si no existiese el derecho a recibir la información. El concepto mismo de información implica que deba existir alguna persona que pueda recibirla, de otro modo seria necesario pensar en un derecho de informar que puede vivir incluso en una especie de "vacío neumático", el cual no es concebible.

El derecho a recibir informaciones se configura y se articula de manera perfectamente paralela y como reflejo respecto al derecho a informar. De igual modo que el primero, este último exige una plena y efectiva libertad de elección respecto a los "medios" a través de los cuales se quiere o se prefiere recibir las informaciones: todos los medios a disposición de la libre elección de quien informa deben igualmente estar a disposición de quien desea recibir la información ofreci-

5 N. del T.: El autor utiliza la palabra italiana specularitá, que no tiene traducción literal al castellano. Se refiere a que el derecho a recibir información es "la otra cara de la moneda" del derecho a informar. 
da. Cada vez que un individuo tenga indicio de la "oferta" de una determinada información, debe poder libremente elegir para su recepción el medio en el cual una determinada información se concreta y materializa: la palabra, la escritura, la imprenta, la radio, la televisión, las nuevas tecnologías de cualquier tipo que sean. Tal derecho a la plena libertad de elección de los medios de recepción, parece implicar la inadmisibilidad de límites, condiciones, obstáculos de la naturaleza que se quiera interpuestos frente a la libertad de elección que se acaba de subrayar. Desde este punto de vista aparece extremadamente dudosa la legitimidad incluso de las condiciones de carácter fiscal a las que se subordina la detentación, y por tanto el libre uso de medios de recepción radiofónicos y televisivos, incluso si esta cuestión fuese analizada en profundidad con relación a particulares aspectos tecnológicos y jurídicos. En todo caso, el derecho a la libre elección y uso de cualquier posible medio de recepción debe representar, de modo absoluto, la regla cuya excepción exige una específica y adecuada prueba contraria.

Por último, a propósito del derecho a recibir informaciones, me parece obligado subrayar que me resulta difícilmente aceptable que el derecho pueda considerarse cualificado y condicionado por la "veracidad" de las informaciones que se desean recibir: una consecuencia semejante se deduce quizá de la fórmula adoptada en la Constitución española. El derecho a recibir información debe existir independientemente de la naturaleza, del contenido, las características y el objeto de la información que se pretende recibir. De igual modo que el derecho a informar existe y tiene sentido independientemente de una presunta "verdad objetiva" de la información misma - como he tratado de demostrar por extenso en la primera parte de mi discurso- otro tanto no puede no aplicarse con respecto a los sujetos del lado "pasivo" de la relación de comunicación informativa: semejante "verdad" no puede representar ni una condición ni un límite para el derecho de quien recibe, y por otro lado no puede representar de ningún modo su pretensión jurídica. Todas mis precedentes afirmaciones se inscriben en la lógica del pleno paralelismo entre el derecho de quien informa y el de quien recibe. El derecho a recibir informaciones tiene un sentido, existe, $y$ tiene objeto, en referencia al si, al cuándo, al cómo, a de qué manera y con qué contenido la información se ofrece en el ámbito de lo que, quizá de modo imaginario, puede ser descrito como el "mercado informativo»: en tal "mercado" todos pueden ofrecer la información que quieran, y todos pueden adquirir las informaciones ofrecidas. Nada más y nada menos. 


\section{EL DERECHO A BUSCAR LA INFORMACIÓN: NECESIDAD DE SU CORRECTA DELIMITACIÓN}

El tercer derecho del que se habla es el de buscar las informaciones. Brevemente, a propósito de tal derecho, es necesario realizar una aclaración muy importante. Efectivamente: éste puede referirse a la búsqueda de las informaciones que son puestas a disposición de (o en todo caso no vedadas a) quien desea buscarlas, o que están de alguna manera disponibles; o por el contrario este derecho se puede referir a la búsqueda de las informaciones que non son puestas a disposición, o cuya búsqueda es excluida o vetada por otros, de cualquier modo y bajo cualquier perfil. El primer aspecto representa el presupuesto más elemental y natural del derecho a recibir las informaciones que sean ofrecidas (o en cualquier caso presentes) en el "mercado" de la comunicación. Es obvio que, para recibir las correspondientes «informaciones", cualquiera debe poder ingeniárselas para tomar conocimiento (hasta el punto de, por así decir, investigar) de los fenómenos y de los eventos que en cualquier manera lo rodean: más específicamente, por ejemplo, para tener acceso a la visión de los datos escritos - publicaciones impresas, para sintonizarse con determinadas emisoras radiotelevisivas, para conectarse con cualquier tipo de fuente informativa o de "sitio" internet, etc. Entendido en tal sentido, el derecho a buscar es (o debería ser) del todo obvio y del todo indispensable, y por tanto también totalmente fundamental e inviolable: el derecho a recibir informaciones no sería ni concebible ni practicable si asimismo no fuese necesariamente garantizado de modo similar el derecho a la búsqueda; esto, entre otras cosas, revela un ulterior perfil bajo el cual se presentan como ilegítimos los límites y los vínculos, incluso de naturaleza fiscal, que de modo más menos intenso compriman el uso o el disfrute de los medios "técnicos" capaces de permitir el ejercicio de la libertad de búsqueda y de recepción.

Cosa bien distinta del aspecto ahora señalado es, sin embargo, el derecho a la búsqueda de informaciones que no se encuentran a disposición o que de alguna manera no están disponibles. Yo tiendo a excluir que jurídicamente exista o sea configurable un derecho semejante ( $y$ por supuesto no debería tener valor constitucional), porque resulta evidente que prescinde de su estrecha vinculación con los derechos específicamente garantizados por la libre relación de comunicación informativa. Si se refiere a actos, actividades, documentos, comportamientos, o similares, el derecho del que hablamos puede ser, todo lo más, previsto por un determinado ordenamiento (pero, habitualmente, no como inviolable derecho constitucional), 
especialmente si éste prevé que los actos, procedimientos, documentos de sujetos públicos $-y$ sólo en cuanto tales- están abiertos al "acceso" de otros sujetos que puedan alegar un interés jurídico para llegar a conocerlos. Incluso en ese caso, sin embargo, se trata de un derecho que no tiene nada que ver con los relativos a la libre relación de comunicación informativa y que, por tanto, es difícil entender que goce de una propia y especifica garantía constitucional. Por lo que se refiere al caso en el cual la búsqueda de informaciones quiera referirse a actos, hechos o comportamientos con relación a los cuales otros sujetos pretendan impedir -legítimamente- su conocimiento, e intenten -siempre legítimamente- evitar su muestra al público, no parece aplicable el correspondiente derecho constitucional. Todo lo más se tratará de una actividad de investigación puramente "de facto", que puede ser como mucho situada en el ámbito del mero "acto lícito", y cuya justificación debe entenderse que desaparece frente a actividades, comportamientos, actos de otros sujetos que puedan presentar, también a nivel constitucional, una esfera protegida de "intimidad" o incluso de "secreto". Ningún derecho constitucional a la búsqueda de informaciones en el sentido indicado, en consecuencia y en ningún caso, me parece configurable: tal conclusión, entre otras cosas, excluye que pueda considerarse verdadero y propio derecho la actividad (al menos en buena medida) desarrollada por el llamado "periodismo de investigación». En concreto, me parece que debe excluirse que semejante actividad pueda jactarse de cualquier forma de "privilegio" derivado de (o vinculado a) un cierto derecho particular, que tendría además nivel constitucional.

\section{EL DERECHO A OBTENER UNA INFORMACIÓN VERDADERA} E IMPARCIAL: DUDAS SOBRE SU CONFIGURABILIDAD. LÍNEAS CRITICAS SOBRE EL LLAMADO «PLURALISMO"; IMPLICACIONES QUE SE DERIVAN DEL CORRECTO MODO DE ENTENDER LA «INFORMACIÓN»

El cuarto de los derechos más arriba indicados debería ser, según algunos, el de recibir, o mejor, obtener una información que posea determinadas características "de contenido" (o "de calidad", si quiere decirse): una información imparcial, completa, "plural" y, en especial, "objetivamente verdadera". Éste, según algunos, sería el nuevo $y$ emergente derecho referible al fenómeno de la información, que se fundaría sobre el "valor" del llamado "pluralismo", y que derivaría de las exigencias típicas y esenciales de toda democracia. 
A mi juicio, sin embargo, un derecho semejante se presenta basado en un fundamento $y$ naturaleza tales que plantean dudas probablemente insuperables sobre su propia configurabilidad. El discurso al respecto se alargaría, y para desarrollarlo adecuadamente sería necesario un tratamiento específico. Debo por ello limitarme a los siguientes apuntes. Con relación a la naturaleza de tal derecho, hasta quien tiende a afirmarlo reconoce que sería un "derecho social" (y por tanto no un verdadero y propio "derecho de libertad"); por eso, en cuanto a su estructura jurídica, se configuraría como un derecho de, o mejor como un derecho a una prestación: sería por tanto un derecho al cual debería necesariamente corresponder alguna forma de obligación, impuesta o garantizada por los poderes públicos, a cargo de los sujetos que proporcionen las informaciones. Precisamente por ésta su naturaleza, concebir (y afirmar) un derecho semejante significa contraponerlo al derecho a informar libremente; el primero de los derechos ahora indicados es, por lo tanto, un derecho tal que entraría en potencial conflicto, más o menos evidente, con el segundo. Afirmar un derecho semejante a obtener "buenas" informaciones significa, por ello, incidir negativamente, de manera más o menos grave, en función de su concreta especificación (pero siempre, en todo caso) sobre la consistencia y las características del clásico, tradicional y fundamental derecho de libertad a informar.

En segundo lugar, la referencia al valor del pluralismo, que en definitiva viene aludido para justificar la afirmación del derecho en cuestión, no puede ser considerada como una prueba. Ya sea por la incerteza y la equivocidad -o al menos, la generalidad- del concepto de pluralismo, ya sea porque -como he dicho al principio- el llamado "pluralismo" no puede representar un presupuesto y parámetro en el ámbito de los derechos referidos o referibles a la información, sino que en todo caso, como mucho, es solamente un resultado de la plena y efectiva libertad de informar. Por último, el derecho que se pretende afirmar se revela como privado de consistencia porque tendría un objeto imposible: si uinformación" es cualquier tipo de "mensaje»; si la llamada "verdad objetiva" no es ni concebible, ni predicable, ni adecuadamente practicable respecto a la información; si toda información es -en el sentido y por las razones precedentemente indicadas - "tendenciosa"; si todo esto es exacto, el pretendido y presunto derecho a obtener una información imparcial, correcta, completa, objetivamente verdadera, se revela ausente de sentido en cuanto a un posible objeto, y por tanto, en sintesis una peligrosa ilusión. 


\section{LÍNEAS CONCLUSIVAS, Y ALGUNAS CONSECUENCIAS DEL DISCURSO DESARROLLADO; \\ EL "MITO» DE LA «INFORMACIÓN" COMO "SERVICIO PÚBLICO»}

He llegado a la conclusión de mi discurso, quizá excesivamente largo. Todas las consideraciones que hasta ahora he intentado desarrollar, tomadas de modo conjunto, me permiten indicar una última consecuencia suya en lo que se refiere a la valoración de la tesis, muy difundida y recurrente con frecuencia en los textos normativos (italianos y de otros países), y sostenida por una parte de los especialistas, según la cual la información sería (por su naturaleza y en cuanto tal) un "servicio público", o al menos lo sería en el caso de los grandes medios de comunicación social: en particular lo sería en el caso de los medios radiofónicos y televisivos. A mi juicio, la tesis de la información como "servicio público», y su consiguiente tratamiento jurídico, es sólo un mito del todo disparatado y capaz de implicaciones - si se lleva a las lógicas consecuencias- muy negativas.

El concepto de "servicio público" de un lado presupone que se pueda poseer certeza suficiente para individuar las características cuantitativas y cualitativas de la actividad que tiene por objeto la prestación del "servicio", y la imposición de obligaciones de conformarse a los consiguientes parámetros; y de otro lado exige intrínsecamente que la prestación en juego deba ser calificada, o en todo caso deba poder ser calificable, como jurídicamente "obligada». Sin embargo, en el caso de la información, si es objeto de verdaderos y propios derechos de libertad, ambas condiciones no son ni posibles ni aceptables. Efectivamente, en mi opinión, la información no posee características y connotaciones específicas identificables con objetividad y neutralidad, y no es admisible calificar como de algún modo "obligado" el "si", el "qué cosa", el cómo y el cuándo de una actividad (la informativa) que, cuando es atribuida e imputada a los individuos en cuanto sujetos "privados", puede ser solamente considerada y tratada como ejercicio de una libertad individual.

El «mito" del servicio público de la, y para la, información es, por tanto, un mito a rechazar $y$ desacreditar. Y con esta afirmación, quizá expeditiva, entiendo que es posible concluir. 